\title{
Intra-arterial treatment of patients with acute ischemic stroke and internal carotid artery occlusion: a literature review
}

\author{
Manon Kappelhof, ${ }^{1}$ Henk A Marquering, ${ }^{1,2}$ Olvert A Berkhemer, ${ }^{1}$ \\ Charles B L M Majoie ${ }^{1}$
}

- Additional material is published online only. To view please visit the journal online (http://dx.doi.org/10.1136/ neurintsurg-2013-011004).

${ }^{1}$ Department of Radiology, Academic Medical Center, University of Amsterdam, Amsterdam, The Netherlands ${ }^{2}$ Department of Biomedical Engineering and Physics, Academic Medical Center, University of Amsterdam, Amsterdam, The Netherlands

Correspondence to Dr Henk A Marquering, Departments of Radiology and Biomedical Engineering and Physics, AMC Amsterdam, Meibergdreef 9, Amsterdam 1105 AZ, The Netherlands; h.a.marquering@amc.uva.nl

Received 11 October 2013 Revised 5 December 2013 Accepted 12 December 2013 Published Online First 2 January 2014

\section{CrossMark}

\footnotetext{
To cite: Kappelhof $M$ Marquering $\mathrm{HA}$, Berkhemer OA, et al. J Neurolntervent Surg 2015;7:8-15.
}

\section{ABSTRACT}

Introduction Acute ischemic stroke (AIS) due to internal carotid artery (ICA) occlusion has a poor prognosis if treated by intravenous thrombolysis. Intra-arterial (IA) treatment is a promising alternative. However, its effectiveness is still unproven.

Methods We collected all publications describing results of IA treatment in acute ICA occlusions. All studies with 10 patients or more providing data on clinical outcome were included. We pooled the results by occlusion site (intracranial, extracranial or tandem occlusions) and treatment method, using recanalization, symptomatic intracranial hemorrhage, mortality and favorable clinical outcome (defined as modified Rankin Scale score 0-2) as outcome measures.

Results 32 studies totaling 1107 patients were included. In the population with intracranial ICA occlusion, recanalization $(69 \%$ vs $38 \%, p<0.001)$ and favorable outcome ( $34 \%$ vs $12 \%, p<0.001)$ rates were significantly higher for mechanical thrombectomy than for IA thrombolysis. In the population with extracranial ICA occlusion, stenting resulted in a higher recanalization rate $(87 \%$ vs $48 \%, p=0.001)$ and favorable outcome rate $(68 \%$ vs $15 \%, p<0.001)$ and lower mortality ( $18 \%$ vs $41 \%, p=0.048)$ than IA thrombolysis. In the tandem occlusion population, a statistically significant lower death rate was found for the group treated with IA thrombolysis only compared with the groups with any mechanical treatment of the intracranial occlusion ( $0 \%$ vs $34 \%, p=0.002$ and $0 \%$ vs $33 \%, p=0.001$ ).

Conclusions This review shows that, for patients with AIS due to an extracranial and/or intracranial ICA occlusion, stenting and mechanical thrombectomy are associated with higher recanalization rates and improved functional outcomes compared with IA thrombolysis.

\section{INTRODUCTION}

Intravenous thrombolysis (IVT) with recombinant tissue plasminogen activator ( $\mathrm{r}-\mathrm{tPA}$ ) is the most common treatment for acute ischemic stroke (AIS). However, results of this treatment are poor in patients with large vessel occlusions. ${ }^{1} 2$ Patients with AIS secondary to an internal carotid artery (ICA) occlusion have an especially poor prognosis. According to a recent study on treatment results for this patient group, there was no significant difference in recanalization or clinical outcome rates between IVT and placebo. ${ }^{3}$ Other studies report that IVT of AIS due to ICA occlusions results in a death rate of $24-27 \%$ of patients. ${ }^{4-6}$ In these studies, good clinical outcome, represented as a modified Rankin Scale (mRS) score of 0-2, occurred in only $10-29 \%$ of cases. ${ }^{4-6}$ It has been suggested that intra-arterial (IA) treatment may be a better alternative for this specific patient population. A recent systematic review ${ }^{7}$ suggests that the endovascular treatment of acute ICA occlusions results in improved clinical outcomes compared with IVT. However, endovascular treatment approaches are heterogeneous and may include IA thrombolysis (IAT), microwire clot disruption (MCD), angioplasty, thromboaspiration, stenting and thrombectomy with stent retrievers or MERCI retrievers.

In this review our aim was to compare the rates of recanalization, symptomatic intracranial hemorrhage (sICH), clinical outcome and mortality of mechanical therapy for intracranial ICA occlusion and stenting for extracranial ICA occlusion with IAT.

\section{METHODS}

The PubMed/Medline database was searched for data from January 1995 through June 2013. We used the search terms 'stroke', 'carotid', 'occlusion', 'thrombolytic therapy', 'endovascular' and their synonyms. For greater specificity, we divided this search into articles that were not (yet) indexed for Medline and articles that had already been indexed. This way we could use the filter 'Humans' on the latter search. A single observer (MK) reviewed all the articles that described the results of IA treatment of ICA occlusions. Only articles in English language were included. Case reports and articles that were not available online were excluded. All articles with fewer than 10 cases of endovascular treatment were excluded to minimize the chance of an anecdotal outcome. If a study also contained patients with an occlusion at a location other than the ICA, we only included the ICA occlusion cases. ICA occlusions caused by a dissection were excluded. We only included studies that reported clinical outcomes beyond 30 days, excluding studies that only described functional outcome and mortality at discharge. Also, studies that included patients with various large vessel occlusions without explicitly reporting the clinical outcome of the patients with ICA occlusions were excluded. The ICA occlusion had to be confirmed by at least one imaging modality such as carotid ultrasound, digital subtraction 
angiography (DSA), MR angiography (MRA) or CT angiography (CTA). This criterion did not exclude any articles. One article was excluded because it did not specify which endovascular treatment was given. Three studies used the data of the MERCI and Multi MERCI trials. ${ }^{8-10}$ Two of these were excluded to prevent double usage of the same data sets. Figure 1 shows a flow diagram presenting the search and inclusion and exclusion of publications identified in the literature review.

The search resulted in 32 articles. Treatment modality was recorded and, if possible, the patient groups were categorized according to ICA occlusion site and treatment. The outcome measures were success of recanalization, sICH, favorable outcome and death rates.

Twenty-six studies used either the Thrombolysis In Myocardial Infarction (TIMI) or Thrombolysis in Cerebrovascular Infarction (TICI) score to describe the success of recanalization. One study used the Mori score, ${ }^{11}$ which is comparable to the TIMI and TICI scores. In the other five studies, definitions based on the judgment of the interventional radiologist such as 'successful' were used. ${ }^{12-16}$ A favorable long-term functional outcome was defined as a mRS score of $0-2$ or a Barthel Index of $>90$, assessed 1 month or later after treatment. Only one study used a combination of the mRS and National Institute of Health Stroke Scale (NIHSS) scores. ${ }^{17}$ Most studies defined sICH as an intracranial hemorrhage with a worsening of 4 points on the NIHSS scale. ${ }^{18}$ In some studies, hemorrhages with clinical symptoms or type II parenchymal hemorrhages were reported. These were considered sICHs as well in this review.

If data were missing from an article, we searched for online supplementary tables. If the missing information was not retrievable but did not concern one of the two primary outcome measures (mortality and long-term functional outcome), this was noted and the study was included. If one of the two primary outcome measures was missing, the study was excluded. In two studies ${ }^{19} 20$ the information was incomplete or unclear. In these cases, we tried to contact the corresponding authors by email. In one study ${ }^{19}$ the data were retrieved in this way. The second study was not excluded but the inconsistency is noted in table 1 .

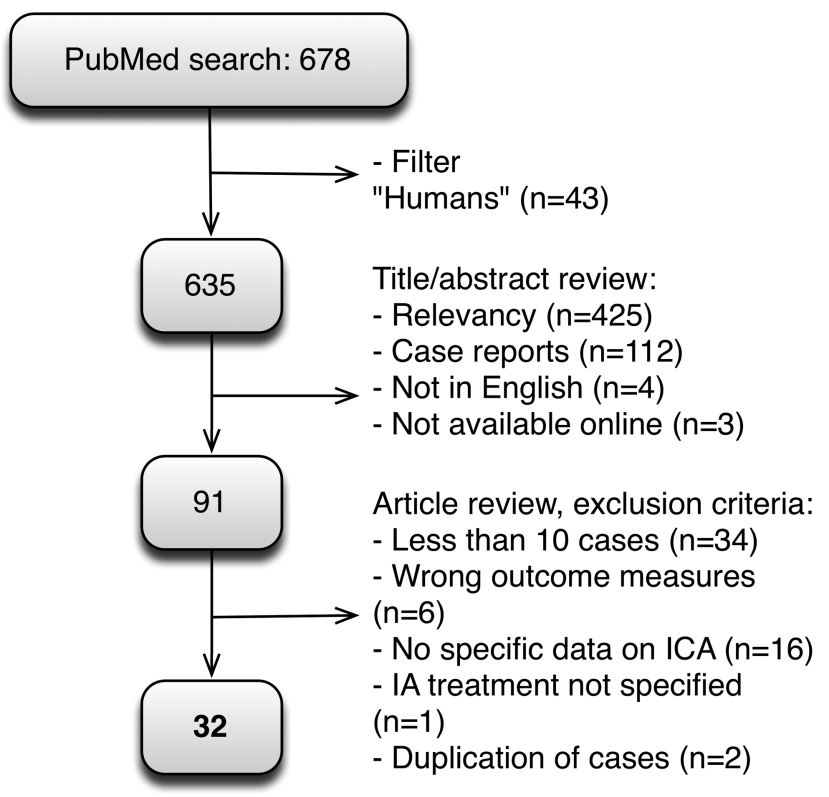

Figure 1 Flowchart showing screening and selection of studies during the search process (n denotes the number of excluded studies). IA, intra-arterial; ICA, internal carotid artery.
After collecting the data, we grouped the results by occlusion sites, creating three populations. Population 1 consisted of patients with an intracranial ICA occlusion, patients in population 2 had an extracranial ICA occlusion and patients in the third population had a tandem occlusion (a simultaneous occlusion of the extracranial and ipsilateral intracranial ICA). We did not discriminate for prior IVT treatment. For patients with an intracranial ICA occlusion (population 1), the treatment options were IAT with or without MCD and mechanical thrombectomy (MT). MT included angioplasty, aspiration thrombectomy (Penumbra) and retrieval with a MERCI device or stent retrievers (Solitaire, Trevo, Revive). For patients with an extracranial ICA occlusion (population 2), the treatment possibilities consisted of IAT with or without MCD and stenting with or without aspiration. For patients with a tandem occlusion (population 3), we analyzed recanalization rates of IAT and MT in intracranial occlusions and of IAT and stenting in extracranial occlusions. Furthermore, we analyzed clinical outcomes for patients with a tandem occlusion. We grouped together studies with similar treatment of extracranial and intracranial occlusion, resulting in four groups: (1) IAT treatment of both extracranial and intracranial occlusions; (2) stenting of the extracranial occlusion and IAT of the intracranial occlusion; (3) stenting of the extracranial occlusion and treatment with stent retrievers of the intracranial occlusion; and (4) stenting of the extracranial occlusion and a combination of multiple treatments for the intracranial occlusion.

SPSS Statistics V.21 was used to perform statistical analysis. Because the outcome measures (recanalization, mortality, sICH and favorable outcome) were dichotomous, we used the $\chi^{2}$ test to calculate the significance levels. A level of $\mathrm{p}<0.05$ was considered significant. For the comparison of the different tandem occlusion groups, the numbers were too low to use the $\chi^{2}$ test so the Fisher test was used instead.

\section{RESULTS}

We included 32 studies with a total of 1107 patients with a mean \pm SD age of $65 \pm 4.9$ years. The overall recanalization rate was $70 \%$ (771), the sICH rate was 9\% (97), the death rate was $33 \%$ (365) and the favorable outcome rate was 29\% (321). Table 1 gives an overview of the collected data presented in chronological order.

\section{Intracranial occlusions (population 1)}

We included six studies ${ }^{412} 14202231$ totaling 95 patients with an intracranial occlusion treated by IAT (see online supplementary table S1) and eight studies ${ }^{17} 20263032363840$ with 115 patients with an intracranial occlusion treated by MT (see online supplementary table S1). Overall outcome rates were $55 \%$ recanalization, $12 \% \mathrm{sICH}, 34 \%$ mortality and $25 \%$ favorable outcome (see online supplementary table S2). Comparison between treatments showed a higher recanalization rate $(69 \%$ vs $38 \%, \mathrm{p}<0.001)$ and favorable outcome rate $(34 \%$ vs $14 \%$, $\mathrm{p}<0.001)$ and a lower death rate $(29 \%$ vs $40 \%, \mathrm{p}=0.085)$ for patients with MT. The sICH rates were similar for both treatments $(12.2 \%$ vs $11.7 \%, \mathrm{p}=0.085$; figure 2$)$.

\section{Extracranial occlusions (population 2)}

Two studies ${ }^{13} 23$ with a total of 27 patients described results of IAT for extracranial ICA occlusions (see online supplementary table S3). The stenting group comprised four studies ${ }^{15} 162435$ and a total of 38 patients (see online supplementary table S3). Overall outcome rates were $71 \%$ recanalization, $2 \% \mathrm{sICH}, 28 \%$ mortality and $46 \%$ favorable outcome (see online 
Table 1 Overall results of IA therapy

\begin{tabular}{|c|c|c|c|c|c|c|c|c|c|c|c|c|}
\hline Study & n & $\begin{array}{l}\text { Mean } \\
\text { age }\end{array}$ & $\begin{array}{l}\text { Mean NIHSS } \\
\text { at intake }\end{array}$ & $\begin{array}{l}\text { Mean TT } \\
\text { (min) }\end{array}$ & Treatment & $\operatorname{Rec}(\%)$ & Definition rec & $\begin{array}{l}\text { sICH } \\
(\%)\end{array}$ & $\begin{array}{l}\text { Mortality } \\
(\%)\end{array}$ & $\begin{array}{l}\text { Favorable } \\
\text { outcome (\%) }\end{array}$ & $\begin{array}{l}\text { Definition of favorable } \\
\text { outcome }\end{array}$ & Occlusion site \\
\hline Urbach et $a l^{12}$ & 12 & 51 & NR & 190 & IAT (UK) & $4(33)$ & Good/moderate & $1(8)$ & $4(33)$ & $4(33)$ & $\mathrm{BI}>90$ at 3 months & $\mathrm{T}$ \\
\hline \multirow[t]{2}{*}{ Endo et al ${ }^{13}$} & 16 & 68 & NR (>24) & $\begin{array}{l}\text { NR } \\
(<360)\end{array}$ & IAT (UK/rtPA) & $3(19)$ & Successful & $1(6)$ & $10(62.5)$ & $0(0)$ & mRS $0-2$ at 1 months & EC \\
\hline & 5 & & & & IAT (UK/rtPA)+PTA/CEA & $5(100)$ & Successful & $0(0)$ & $0(0)$ & $4(80)$ & mRS $0-2$ at 1 months & \\
\hline Zaidat et $a l^{14}$ & $\begin{array}{l}5 \\
13\end{array}$ & 69 & $\begin{array}{l}15^{*} \\
15^{*}\end{array}$ & $\begin{array}{l}143 \\
175\end{array}$ & $\begin{array}{l}\text { IAT (UK)+IVT (rtPA) } \\
\text { IAT (UK) }\end{array}$ & $\begin{array}{l}4(80) \\
8(62)\end{array}$ & $\begin{array}{l}\text { Partial/complete } \\
\text { Partial/complete }\end{array}$ & $\begin{array}{l}1(20) \\
2(15)\end{array}$ & $\begin{array}{l}1(20) \\
8(62)\end{array}$ & $\begin{array}{l}3(60) \\
3(23)\end{array}$ & $\begin{array}{l}\text { mRS } 0-2 \text { at } 3 \text { months } \\
\text { mRS } 0-2 \text { at } 3 \text { months }\end{array}$ & $\begin{array}{l}3 \mathrm{~T}, 2 \mathrm{~L} \\
7 \mathrm{~T}, 4 \mathrm{~L}, 2 \text { cervical }\end{array}$ \\
\hline Arnold et $a^{21}$ & 24 & 60 & $19^{*}$ & 237 & IAT (UK) \pm angioplasty, stent & $15(62.5)$ & TIMI $2 / 3$ & $1(4)$ & $10(42)$ & $4(17)$ & mRS $0-2$ at 3 months & $T$ \\
\hline Eckert et $a l^{22}$ & 43 & 55 & $\mathrm{NR}(>9)$ & $\begin{array}{l}\text { NR } \\
(<360)\end{array}$ & IAT (UK/rtPA/rtPA+lys-plasminogen) & $13(30)$ & TIMI 2/3 & NR & $15(35)$ & $6(14)$ & $\mathrm{BI}>90$ at 3 months & $35 \mathrm{~T}, 8$ tandem \\
\hline Sorimachi et $a l^{23}$ & 11 & 71 & 23 & 212 & IAT (UK)+MCD & $10(91)$ & Opened & $0(0)$ & 1 (9) & $4(36)$ & mRS $0-2$ at 3 months & $\mathrm{EC}$ \\
\hline Jovin et $a l^{24}$ & 15 & 59 & 16 & 300 & Stent+angioplasty+8 IVT (rtPA) & $15(100)$ & Successful & $0(0)$ & $3(20)$ & $5(33)$ & mRS $0-2$ at 1 months & $5 \mathrm{EC}, 10$ tandem \\
\hline $\begin{array}{l}\text { Nedeltchev } \\
\text { et } a l^{25}\end{array}$ & 25 & 59 & $12^{*}$ & 169 & $\begin{array}{l}\text { Stent+IAT } \\
(\text { UK) } \pm \text { aspiration }\end{array}$ & $21(84)$ & TIMI 2/3 & $2(8)$ & $5(20)$ & $14(56)$ & mRS $0-2$ at 3 months & $21 \mathrm{EC}, 4 \mathrm{~T}$ \\
\hline Mori et $a l^{26}$ & 14 & 76 & 20 & 183 & Stent retrieval+aspiration & $7(50)$ & TIMI 2/3 & $0(0)$ & $2(14)$ & $6(43)$ & mRS $0-2$ at 3 months & $6 \mathrm{EC}, 8 \mathrm{IC}$ \\
\hline Suh et $a l^{27}$ & 30 & 66 & $15^{*}$ & NR & $\begin{array}{l}16 \text { IAT, } 9 \text { IAT+stenting/thrombectomy, } 3 \\
\text { IAT+angioplasty, } 2 \text { stent only }\end{array}$ & $14(47)$ & $\mathrm{TICl} 2 / 3$ & $9(30)$ & $6(20)$ & $11(37)$ & mRS $0-2$ at 1 year & $26 \mathrm{EC}, 7 \mathrm{IC}$ \\
\hline Arnold et $a^{28}$ & 43 & 60 & NR & 257 & IAT (UK) & $26(58)$ & TIMI 2/3 & $4(9)$ & $16(37)$ & $10(23)$ & mRS $0-2$ at 3 months & $30 \mathrm{~T}, 13 \mathrm{EC}$ \\
\hline $\begin{array}{l}\text { Miyamoto } \\
\text { et } \mathrm{al}^{15}\end{array}$ & 10 & 77 & 11 & 330 & $\begin{array}{l}\text { Stent+angioplasty+IVT (UK)+for } \\
\text { intracranial IAT (UK) }\end{array}$ & $10(100)$ & Complete & $0(0)$ & $1(10)$ & $6(60)$ & mRS $0-2$ at 3 months & $5 \mathrm{EC}, 5$ tandem \\
\hline Kim et al ${ }^{29}$ & 10 & 60 & 13 & 289 & IAT (UK) & $6(60)$ & TIMI 2/3 & $1(10)$ & $0(0)$ & $5(50)$ & mRS $0-2$ at 3 months & Tandem \\
\hline \multirow[t]{3}{*}{ Lin et $a l^{20} \dagger$} & 40 & 68 & $17^{*}$ & 343 & $\begin{array}{l}\text { IAT (rtPA/UK)+mechanical (MERCI } \\
\text { retrieval/angioplasty/stent) }\end{array}$ & $26(65)$ & TIMI 2/3 & $3(7.5)$ & $14(35)$ & $17(23)$ & mRS $0-2$ at 3 months & $61 \mathrm{~T}, 14 \mathrm{~L}$ \\
\hline & 18 & 71 & $18^{*}$ & 374 & $\begin{array}{l}\text { Mechanical (MERCI retriever/ } \\
\text { angioplasty/stent) }\end{array}$ & $6(33)$ & TIMI 2/3 & $3(17)$ & $8(44)$ & & mRS $0-2$ at 3 months & \\
\hline & 17 & 63 & $17^{*}$ & 338 & IAT (rtPA/UK) & $3(18)$ & TIMI 2/3 & $2(12)$ & $6(35)$ & & mRS $0-2$ at 3 months & \\
\hline Yoo et $a l^{11}$ & 16 & 69 & 18 & 246 & $\begin{array}{l}\text { IAT (UK)+IVT (rtPA)/MERCI retriever/ } \\
\text { MCD/angioplasty/stent }\end{array}$ & $12(75)$ & Mori 2-4 & $1(6)$ & $5(31)$ & $2(13)$ & mRS $0-2$ at 3 months & $11 \mathrm{~T}, 5 \mathrm{~L}$ \\
\hline \multirow[t]{2}{*}{ Shi et al ${ }^{10}$} & 18 & 68 & 20 & 258 & $\begin{array}{l}\text { Merci retriever+IVT }(r \mathrm{rPA}) \pm \mid \mathrm{IAT}(\mathrm{rtPA} / \\
\text { UK/tenecteplase) }\end{array}$ & $12(67)$ & TIMI 2/3 & $2(11)$ & $6(33)$ & $5(28)$ & mRS $0-2$ at 3 months & $1 \mathrm{EC}, 17 \mathrm{~T}$ \\
\hline & 81 & 68 & 20 & & $\begin{array}{l}\text { Merci retriever } \pm \mid A T \text { (rtPA/UKI } \\
\text { tenecteplase) }\end{array}$ & $50(62)$ & TIMI 2/3 & $9(11)$ & $41(51)$ & $23(28)$ & mRS $0-2$ at 3 months & $27 \mathrm{EC}, 54 \mathrm{~T}$ \\
\hline Miteff et $\left.a\right|^{30}$ & $\begin{array}{l}6 \\
5\end{array}$ & $\begin{array}{l}70 \\
65\end{array}$ & $\begin{array}{l}20 \\
16\end{array}$ & $\begin{array}{l}389 \\
313\end{array}$ & $\begin{array}{l}\text { Stent retrieval } \\
\text { Stent retrieval+Penumbra/MERCI/IAT } \\
\text { (UK) }\end{array}$ & $\begin{array}{l}6(100) \\
4(80)\end{array}$ & $\begin{array}{l}\text { TIMI 2/3 } \\
\text { TIMI 2/3 }\end{array}$ & $\begin{array}{l}0(0) \\
1(20)\end{array}$ & $\begin{array}{l}0(0) \\
1(20)\end{array}$ & $\begin{array}{l}3(50) \\
3(60)\end{array}$ & $\begin{array}{l}\text { mRS } 0-2 \text { at } 3 \text { months } \\
\text { mRS } 0-2 \text { at } 3 \text { months }\end{array}$ & $\begin{array}{l}3 \text { tandem, } 3 \mathrm{~T} \\
2 \mathrm{~T}, 2 \text { tandem, } 1 \\
\mathrm{EC}\end{array}$ \\
\hline Costalat et $a l^{17}$ & 14 & 70 & 15 & 243 & Stent retriever \pm IVT (warfarin) & $10(71)$ & $\mathrm{TICl} 3$ & $0(0)$ & $2(14)$ & $6(43)$ & $\begin{array}{l}\text { mRS } 0-2 \text { at } 3 \text { months/ } \\
\text { NIHSS } 0-1 \text { at } 3 \text { months }\end{array}$ & $\mathrm{T} / \mathrm{L}$ \\
\hline $\begin{array}{l}\text { Watanabe } \\
\text { et }\left.a\right|^{31}\end{array}$ & 10 & 81 & $18^{*}$ & 148 & IAT $(\mathrm{UK}) \pm \mathrm{MCD}$ & $5(50)$ & $\mathrm{TICl} 2 / 3$ & $2(20)$ & $4(40)$ & $1(10)$ & mRS $0-2$ at 3 months & T \\
\hline Fesl et $a l^{32}$ & 14 & 59 & $19^{*}$ & 252 & Aspiration \pm other mechanical & $11(79)$ & TIMI 2/3 & $7(50)$ & $6(43)$ & $3(21)$ & mRS $0-2$ at 3 months & $\mathrm{T}$ \\
\hline $\begin{array}{l}\text { Papanagiotou } \\
\text { et }\left.a\right|^{33}\end{array}$ & 22 & 65 & 17 & 246 & Stent \pm angioplasty \pm IVT $(\mathrm{rtPA})$ & $21(95)$ & TIMI 2/3 & $4(18)$ & $3(14)$ & $9(41)$ & mRS $0-2$ at 3 months & $4 \mathrm{EC}, 18$ tandem \\
\hline Malik et $\left.a\right|^{34}$ & 77 & 63 & 15 & 433 & Stent \pm angioplasty & $58(75)$ & TIMI 2/3 & $8(10)$ & $19(25)$ & $32(42)$ & mRS $0-2$ at 3 months & Tandem \\
\hline Hauck et $a /^{35}$ & 22 & 65 & 14 & NR & Stent \pm IVT $(r+P A)$ & $17(77)$ & TIMI 2/3 & $0(0)$ & $7(32)$ & $11(50)$ & mRS $0-2$ at 3 months & EC \\
\hline Pagola et al $1^{19}$ & $\begin{array}{l}41 \\
29\end{array}$ & $\begin{array}{l}70 \\
71\end{array}$ & $\begin{array}{l}19 \\
18\end{array}$ & $\begin{array}{l}290 \\
300\end{array}$ & $\begin{array}{l}\text { IAT }(r t P A) \pm M C D \\
\text { Stent retriever }\end{array}$ & $\begin{array}{l}25(61) \\
24(83)\end{array}$ & $\begin{array}{l}\mathrm{TICl} 2 / 3 \\
\mathrm{TICI} 2 / 3\end{array}$ & $\begin{array}{l}4(10) \\
3(10)\end{array}$ & $\begin{array}{l}18(44) \\
8(28)\end{array}$ & $\begin{array}{l}5(12) \\
6(31)\end{array}$ & $\begin{array}{l}\text { mRS } 0-2 \text { at } 3 \text { months } \\
\text { mRS } 0-2 \text { at } 3 \text { months }\end{array}$ & NR \\
\hline
\end{tabular}


Table 1 Continued

\begin{tabular}{|c|c|c|c|c|c|c|c|c|c|c|c|c|}
\hline Study & $\mathbf{n}$ & $\begin{array}{l}\text { Mean } \\
\text { age }\end{array}$ & $\begin{array}{l}\text { Mean NIHSS } \\
\text { at intake }\end{array}$ & $\begin{array}{l}\text { Mean TT } \\
\text { (min) }\end{array}$ & Treatment & $\operatorname{Rec}(\%)$ & Definition rec & $\begin{array}{l}\text { SICH } \\
(\%)\end{array}$ & $\begin{array}{l}\text { Mortality } \\
(\%)\end{array}$ & $\begin{array}{l}\text { Favorable } \\
\text { outcome (\%) }\end{array}$ & $\begin{array}{l}\text { Definition of favorable } \\
\text { outcome }\end{array}$ & Occlusion site \\
\hline & 2 & 71 & 21 & 330 & Combined & $2(100)$ & $\mathrm{TICl} 2 / 3$ & $0(0)$ & $1(50)$ & $1(50)$ & mRS $0-2$ at 3 months & \\
\hline Hwang et al ${ }^{36}$ & $\begin{array}{l}20 \\
19\end{array}$ & $\begin{array}{l}65 \\
66\end{array}$ & $\begin{array}{l}18 \\
16\end{array}$ & 127 & $\begin{array}{l}\text { Aspiration } \\
M C D \pm 8 \text { IAT (UK) }\end{array}$ & $\begin{array}{r}17(85) \\
6(32)\end{array}$ & $\begin{array}{l}\mathrm{TICl} 2 / 3 \\
\mathrm{TICl} 2 / 3\end{array}$ & $\begin{array}{l}3(15) \\
2(10.5)\end{array}$ & $\begin{array}{l}0(0) \\
2(10.5)\end{array}$ & $\begin{array}{l}9(45) \\
3(16)\end{array}$ & $\begin{array}{l}\text { mRS } 0-2 \text { at } 3 \text { months } \\
\text { mRS } 0-2 \text { at } 3 \text { months }\end{array}$ & $\mathrm{T} / \mathrm{L}$ \\
\hline Fischer et $a l^{37}$ & $\begin{array}{l}32 \\
78\end{array}$ & 62 & 17 & 280 & $\begin{array}{l}\text { IAT }(\mathrm{UK}) \pm \text { IVT (rtPA) } \\
\text { Mechanical (angioplasty/MCD/stent } \\
\text { retrievers/stents/aspiration) } \pm \text { IVT (rtPA) } \\
\text { IAT (UK)+mechanical } \pm \text { IVT (rtPA) }\end{array}$ & $\begin{array}{l}15(47) \\
64(82)\end{array}$ & $\begin{array}{l}\text { TIMI 2/3 } \\
\text { TIMI 2/3 }\end{array}$ & $\begin{array}{l}1(3) \\
5(6)\end{array}$ & $\begin{array}{l}10(31) \\
24(31)\end{array}$ & $\begin{array}{l}5(16) \\
23(29)\end{array}$ & $\begin{array}{l}\text { mRS } 0-2 \text { at } 3 \text { months } \\
\text { mRS } 0-2 \text { at } 3 \text { months }\end{array}$ & $\begin{array}{l}107 \mathrm{~T}, 76 \text { tandem, } \\
18 \mathrm{EC}, 5 \mathrm{IC}\end{array}$ \\
\hline Yoon et $\left.a\right|^{38}$ & 26 & 73 & 13 & 240 & $\begin{array}{l}\text { Stent-retriever } \pm \text { IAT (UK)/MCD/ } \\
\text { angioplasty/aspiration } \pm \text { IVT (rtPA) }\end{array}$ & $20(77)$ & $\mathrm{TICl} 2 \mathrm{~b} / 3$ & $0(0)$ & $10(38)$ & $10(38)$ & mRS $0-2$ at 3 months & IC \\
\hline Mpotsaris et $a \beta^{39}$ & 41 & 62 & 16 & NR & $\begin{array}{l}\text { Stent retriever } \pm \text { stent (Wallstent) } \pm 34 \text { IVT } \\
\text { (rtPA). }\end{array}$ & $35(85)$ & TIMI 2/3 & $3(7)$ & $15(37)$ & $15(37)$ & mRS $0-2$ at 3 months & Tandem \\
\hline \multirow[t]{2}{*}{ Lee et $a^{40}$} & 10 & 69 & 18 & NR & $\begin{array}{l}\text { Stent retriever+aspiration+IVT (rtPA) } \\
\pm \text { stent }\end{array}$ & $6(60)$ & $\mathrm{TICl} 2 / 3$ & $0(0)$ & $4(40)$ & $0(0)$ & mRS $0-2$ at 3 months & $\mathrm{T} / \mathrm{L}$ \\
\hline & 16 & 66 & 16 & NR & IAT (UK) \pm 7 IVT (tPA) & $12(75)$ & $\mathrm{TICl} 2 / 3$ & 1 (6) & $8(50)$ & $1(6)$ & mRS $0-2$ at 3 months & \\
\hline $\begin{array}{l}\text { Matsubara } \\
\text { et }\left.a\right|^{41} \ddagger\end{array}$ & 14 & 68 & 16 & 175 & Stent/angioplasty/aspiration \pm IVT (tPA) & $14(100)$ & $\mathrm{TICl} 2 / 3$ & $1(7)$ & $4(29)$ & $4(29)$ & mRS $0-2$ at 3 months & 10 tandem, $6 \mathrm{EC}$ \\
\hline Dalyai et al ${ }^{16}$ & 17 & 62 & 17 & NR & Stent+angioplasty \pm IVT (rtPA) & $16(94)$ & 'Successful' & $3(18)$ & $9(53)$ & 9 (53) & mRS $0-2$ at 3 months & $6 \mathrm{EC}, 11$ tandem \\
\hline Kwak et al/2 & 35 & 65 & $12^{*}$ & 450 & Stent+angioplasty \pm 8 IV rtPA & $35(100)$ & $\mathrm{TICl} 2 / 3$ & $1(3)$ & $22(63)$ & $4(11)$ & mRS $0-2$ at 3 months & Tandem \\
\hline
\end{tabular}

Data in italics are extrapolated.

"Data presented as median.

tFavorable outcome rates were only reported for entire patient group, not for each treatment.

¥Originally there were 16 patients, but in two cases the occlusion was caused by a dissection of the ICA. They were excluded and the reported percentages were used for the 14 remaining cases.

ACA, anterior cerebral artery; BI, Barthel Index; CEA, carotid endarterectomy; EC, extracranial; IAT, intra-arterial thrombolysis; IC, intracranial; ICA, internal carotid artery; L, L-occlusion; MCA, medial cerebral artery; MCD, mechanical clot disruption; mRS, modified Rankin Scale; NIHSS, National Institutes of Health Stroke Scale; NR, not retrievable; prox, proximal; PTA, percutaneous transluminal angioplasty; rec, recanalization; rtPA, recombinant tissue plasminogen activator; sICH, symptomatic intracranial hemorrhage; T, T-occlusion; thrombus extending in both the ACA and MCA; thrombus extending in either the ACA or MCA; TICI, Thrombolysis In Cerebral Infarction; TIMI, Thrombolysis In Myocardial Infarction; TT, time to treatment; UK, urokinase. 


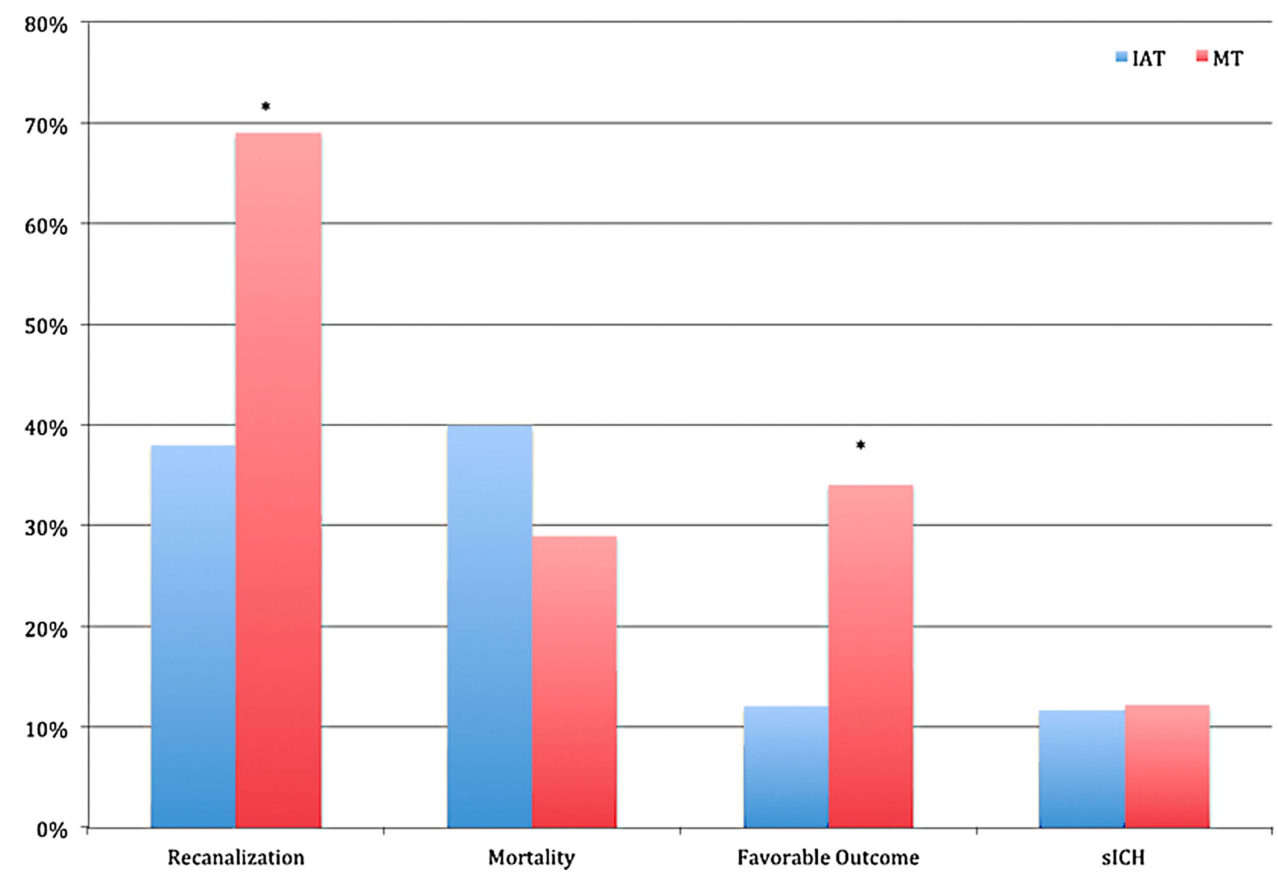

Figure 2 Intra-arterial treatments for intracranial occlusions. ${ }^{*} \mathrm{p}<0.05$. sICH, symptomatic intracranial hemorrhage.

supplementary table S4). The pooled outcome of the treatment results in the extracranial occlusion population is shown in figure 3. Recanalization and favorable outcome rates were significantly higher for stenting than for thrombolysis $(87 \%$ vs $48 \%(\mathrm{p}=0.001)$ and $68 \%$ vs $15 \%(\mathrm{p}<0.001)$, respectively $)$. Mortality was higher for IAT than for stenting, although the difference was only marginally significant $(41 \%$ vs $18 \%$, $\mathrm{p}=0.048)$. The sICH rates were not significantly different $(4 \%$ for IAT, $0 \%$ for stenting, $\mathrm{p}=0.23$ ).

\section{Tandem occlusions (population 3)}

Ten studies with tandem ICA occlusions were included (see online supplementary table S5). Figure 4 shows an overview of the treatment results. Overall outcome rates were $16 \%$ sICH, $45 \%$ mortality and $43 \%$ favorable outcome. We included three studies $^{15} 24{ }^{29}$ with intracranial IAT, comprising a total of 25 patients. Of this population, 15 patients $(60 \%)$ showed sufficient recanalization. Two studies ${ }^{33} 39$ were included that treated the intracranial occlusion with MT. Of this population of 59

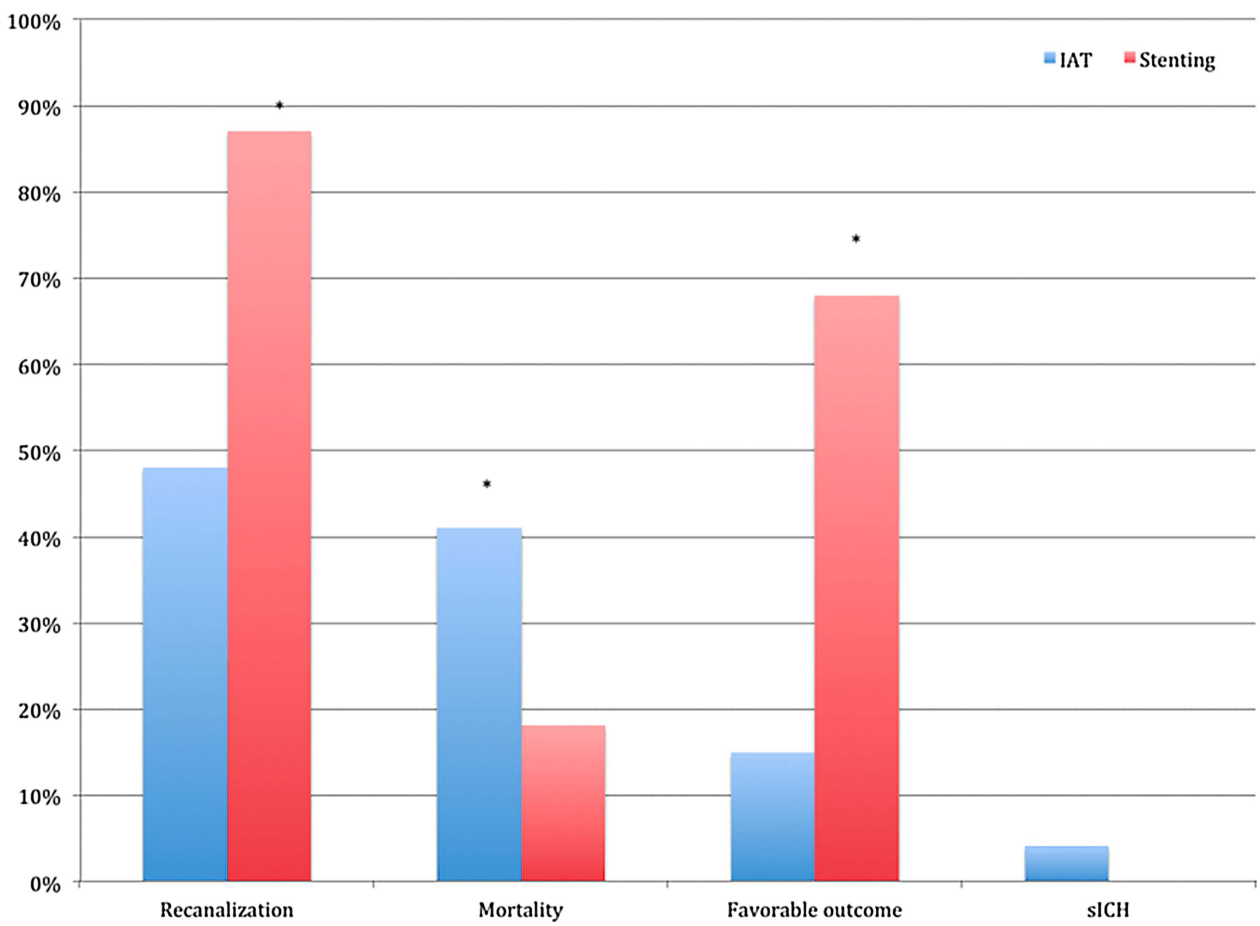

Figure 3 Comparison of outcomes for IAT and stenting in extracranial occlusions. * $p<0.05$. IAT, intra-arterial thrombolysis; sICH, symptomatic intracranial hemorrhage. 


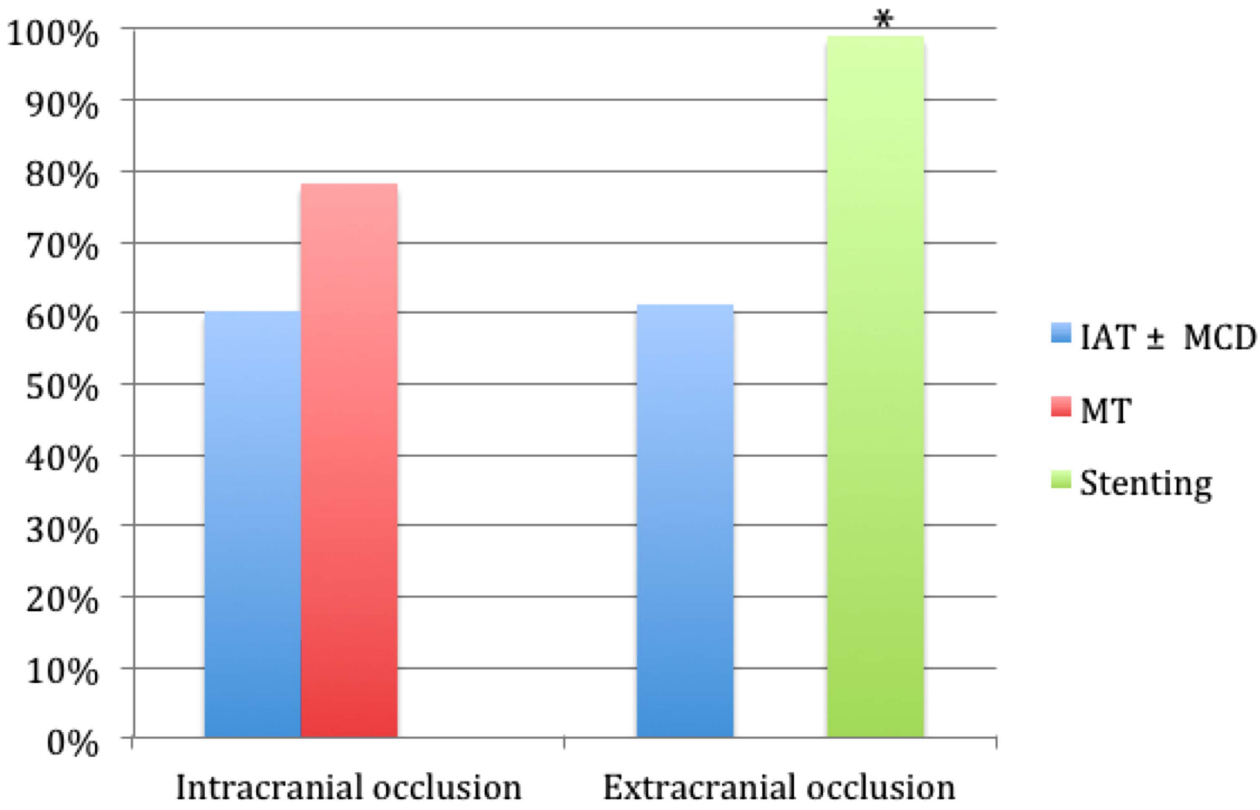

Figure 4 Tandem recanalization rates for intra-arterial thrombolysis (IAT) and mechanical thrombectomy (MT) in intracranial occlusions and IAT and stenting in extracranial occlusions. ${ }^{*} \mathrm{p}<0.05$. MCD, mechanical clot disruption.

patients, 46 showed recanalization $(78 \%)$. This difference in recanalization between IAT and MT was not statistically significant $(\mathrm{p}=0.09)$.

Two studies 22 with a total of 18 patients described IAT of an extracranial ICA occlusion. Eleven patients (61\%) recanalized. Seven studies ${ }^{15} \quad 16 \quad 24 \quad 33 \quad 34 \quad 39 \quad 42$ with 197 patients described stenting of the extracranial ICA occlusion. Of these, 196 (99\%) recanalized resulting in a statistically significant difference in recanalization rate compared with patients treated with IAT of the extracranial ICA $(\mathrm{p}<0.001)$.

In the comparison of the four groups based on intracranial and extracranial treatment, the only statistically significant differences were found for death rates in favor of the combined IAT treatment for both extracranial and intracranial treatment compared with patients with any mechanical treatment of the intracranial occlusion (see online supplementary table S6). These differences were $0 \%$ vs $34 \%(p=0.002)$ for the comparison of IAT for both occlusions with stenting for the extracranial occlusion and stent retrieval for the intracranial occlusion, and $0 \%$ vs $33 \%(p=0.001)$ for the comparison of IAT for both occlusions with stenting for the extracranial occlusion and a combination of IAT and mechanical methods for the intracranial occlusion.

\section{DISCUSSION}

The results of this review suggest that mechanical treatment methods-particularly stenting in extracranial occlusionsachieve better recanalization, higher favorable outcome rates and lower death rates than IAT only in patients with AIS resulting from an occlusion of the ICA.

This study is one of the first to review the IA treatment of AIS due to ICA occlusion. Our review included nine extra studies from 2013 compared with a previous review, ${ }^{7}$ which included articles published only until November 2011, resulting in 406 more patients. Moreover, to our knowledge, this is the first review to differentiate between IA treatment modalities and occlusion site.
For intracranial ICA occlusions, MT was superior to IAT. A possible explanation could be that an occlusion of the ICA is associated with a higher clot burden which makes it more likely for mechanical therapies to achieve recanalization success. Additionally, the mechanical therapies are faster, which may influence the clinical outcome. For extracranial ICA occlusions, stenting was superior to IAT. This might be because the vast majority of extracranial occlusions relate to local severe atherosclerotic plaque. Greater and immediate flow restoration from stenting improves distal perfusion and may deliver endogenous fibrinolytics.

Depending on the configuration of the circle of Willis and associated hemodynamic compromise, acute occlusion of the extracranial ICA may or may not cause symptoms and, as such, patients with an occlusion of the extracranial artery may differ from those with AIS due to an intracranial occlusion. However, extracranial occlusions are treated in the acute setting by some centers. We therefore decided to include patients with acute stroke with purely extracranial ICA occlusions.

Our results are in line with the findings of Mokin et $a l^{7}$ who found a favorable outcome rate of $33.6 \%$ (vs $29.0 \%$ in this review) and a death rate of $32.0 \%$ (vs $33.0 \%$ in this review) for IA treatment. Mokin et al also pooled their results by occlusion site: cervical ICA occlusions (193 patients) and carotid T-occlusions. In patients with a cervical ICA occlusion they found an overall favorable outcome rate of $43.5 \%$, which is in line with the favorable outcome rate of $46 \%$ in the extracranial population in this review. For carotid T-occlusions, Mokin et al reported an overall favorable outcome rate of $28.5 \%$ in the IA treated group, which is also comparable to the mean favorable outcome rate of $24.8 \%$ in our intracranial occlusion population.

A major limitation in this field of research is the lack of large randomized controlled trials (RCTs). Most of our included studies were retrospective so they were prone to selection and other biases. Only a few studies were prospective. In addition, we used both single-center and multicenter studies. This heterogeneity may cause differences in outcome attributable to other factors such as different treatment protocols. Another limitation 
of our included studies is the fact that the choice of the material and techniques used for MT was not prescribed by the trial protocol, but left to the treating interventional radiologist. Moreover, devices are constantly developed and improved over time. Recent RCTs such as the TREVO 2 and SWIFT trials ${ }^{43} 44$ have shown that the recanalization rates and outcomes of stent retrievers are better than those of MERCI retrievers. Therefore, it can be expected that the older studies that used MERCI devices may have had worse recanalization rates and outcomes, influencing the total results of the analysis of the intracranial occlusion group. Finally, another aspect of heterogeneity lies in the fact that the definition of successful recanalization varied between studies. This may have caused falsely low or high recanalization rates.

In our review we limited our evaluation to endovascular treatment modality and its relationship with outcome. Other factors such as the use of IVT and clinical (age, stroke severity, time to treatment, time to recanalization) and imaging parameters (pretreatment ASPECTS, infarct core volume and collateral flow) were not evaluated. By including a large number of studies in our review, the effect of these parameters is probably equally distributed. Nevertheless, the pooling resulted in a few small groups where these other parameters may have had an important impact on clinical outcome.

For the analysis of mortality, favorable outcome and sICH rates in the population with tandem occlusions, the number of the patients per group was low. Only a statistically significant difference was found in the death rate in favor of IAT. However, only two studies were used in this pooling and this difference may also be caused by different patient selection. For example, patients in the IAT/IAT group were, on average, 3-4 years younger than those of the groups with a significantly higher mortality.

Finally, not all studies reported data on occlusion sites or treatment results of different occlusion sites. Those studies could not be included in the pooling of our results. As a result, some of the groups were rather small. This may have biased the pooled outcomes.

We believe that subgroup analysis of patients with ICA occlusion from the recent completed and ongoing trials will improve the evaluation of different IA treatment options.

\section{CONCLUSIONS}

Our review suggests that, in patients with AIS due to ICA occlusion, mechanical approaches are associated with higher recanalization rates and improved functional outcomes compared with IAT.

Contributors CBLMM and HAM contributed to the study design and methodology. MK contributed to the data collection, data interpretation and statistical analysis. HAM, OAB, CBMLM contributed to the data interpretation. HAM contributed to the statistical analysis. MK, HAM, OAB and CBLMM contributed to the Introduction, Methods, Results and Discussion sections, both in writing and revising the manuscript. All authors approved the final version to be published and all authors agreed to be accountable for all aspects of the work.

Competing interests None.

Provenance and peer review Not commissioned; externally peer reviewed.

Data sharing statement Any unpublished data from this study are available through contacting the corresponding author. The authors will be happy to supply any information needed. This includes the PubMed search, the data extraction document, the data calculation Excel document, SPSS files and the Fisher test Excel document.

\section{REFERENCES}

1 Von Kummer R, Hacke W. Safety and efficacy of intravenous tissue plasminogen activator and heparin in acute middle cerebral artery stroke. Stroke 1992;23:646-52
2 Lindsberg PJ, Mattle HP. Therapy of basilar artery occlusion: a systematic analysis comparing intra-arterial and intravenous thrombolysis. Stroke 2006;37:922-8

3 De Silva D, Brekenfeld C, Ebinger $M$, et al. The benefits of intravenous thrombolysis relate to the site of baseline arterial occlusion in the Echoplanar Imaging Thrombolytic Evaluation Trial (EPITHET). Stroke 2010;41:295-9.

4 Kimura K, Iguchi Y, Shibazaki K, et al. Recanalization of the MCA should play an important role in dramatic recovery after t-PA therapy in patients with ICA occlusion. J Neuro/ Sci 2009:285:130-3.

5 Paciaroni M, Agnelli G, Caso V, et al. Intravenous thrombolysis for acute ischemic stroke associated to extracranial internal carotid artery occlusion: the ICARO-2 study. Cerebrovasc Dis 2012:34:430-5.

6 Rubiera M, Ribo M, Delgado-Mederos R, et al. Tandem internal carotid artery/ middle cerebral artery occlusion: an independent predictor of poor outcome after systemic thrombolysis. Stroke 2006;37:2301-5.

7 Mokin M, Kass-Hout T, Kass-Hout O, et al. Intravenous thrombolysis and endovascular therapy for acute ischemic stroke with internal carotid artery occlusion: a systematic review of clinical outcomes. Stroke 2012:43:2362-8.

8 Flint AC, Duckwiler GR, Budzik RF, et al. Mechanical thrombectomy of intracranial internal carotid occlusion: pooled results of the MERCI and Multi MERCI Part I trials. Stroke 2007;38:1274-80

9 Nogueira RG, Liebeskind DS, Sung G, et al. Predictors of good clinical outcomes, mortality, and successful revascularization in patients with acute ischemic stroke undergoing thrombectomy: pooled analysis of the Mechanical Embolus Removal in Cerebral Ischemia (MERCI) and Multi MERCI Trials. Stroke 2009;40:3777-83.

10 Shi Z-S, Loh Y, Walker G, et al. Endovascular thrombectomy for acute ischemic stroke in failed intravenous tissue plasminogen activator versus non-intravenous tissue plasminogen activator patients: revascularization and outcomes stratified by the site of arterial occlusions. Stroke 2010;41:1185-92.

11 Yoo AJ, Verduzco L, Schaefer PW, et al. MRI-based selection for intra-arterial stroke therapy: value of pretreatment diffusion-weighted imaging lesion volume in selecting patients with acute stroke who will benefit from early recanalization. Stroke 2009:40:2046-54.

12 Urbach $\mathrm{H}$, Ries $\mathrm{F}$, Ostertun $\mathrm{B}$, et al. Local intra-arterial fibrinolysis in thromboembolic " $T$ " occlusions of the internal carotid artery. Neuroradiology 1997;39:105-10.

13 Endo S, Kuwayama N, Hirashima Y, et al. Results of urgent thrombolysis in patients with major stroke and atherothrombotic occlusion of the cervical internal carotid artery. AJNR Am J Neuroradiol 1998;19:1169-75.

14 Zaidat 00, Suarez Jl, Santillan C, et al. Response to intra-arterial and combined intravenous and intra-arterial thrombolytic therapy in patients with distal internal carotid artery occlusion. Stroke 2002;33:1821-7.

15 Miyamoto N, Naito I, Takatama S, et al. Urgent stenting for patients with acute stroke due to atherosclerotic occlusive lesions of the cervical internal carotid artery. Neurol Med Chir 2008;48:49-55.

16 Dalyai RT, Chalouhi N, Singhal S, et al. Stent-assisted endovascular recanalization of extracranial internal carotid artery occlusion in acute ischemic stroke. World Neurosurg 2013;79:143-8.

17 Costalat V, Machi P, Lobotesis K, et al. Rescue, combined, and stand-alone thrombectomy in the management of large vessel occlusion stroke using the solitaire device: a prospective 50-patient single-center study: timing, safety, and efficacy. Stroke 2011:42:1929-35.

18 Seet RCS, Rabinstein AA. Symptomatic intracranial hemorrhage following intravenous thrombolysis for acute ischemic stroke: a critical review of case definitions. Cerebrovasc Dis 2012;34:106-14.

19 Pagola J, Rubiera M, Flores A, et al. Selecting endovascular treatment strategy according to the location of intracranial occlusion in acute stroke. Cerebrovasc Dis 2013;35:502-6

20 Lin R, Vora N, Zaidi S, et al. Mechanical approaches combined with intra-arterial pharmacological therapy are associated with higher recanalization rates than either intervention alone in revascularization of acute carotid terminus occlusion. Stroke 2009;40:2092-7

21 Arnold M, Nedeltchev K, Mattle HP, et al. Intra-arterial thrombolysis in 24 consecutive patients with internal carotid artery T occlusions. J Neurol Neurosurg Psychiatry 2003:74:739-42.

22 Eckert B, Kucinski T, Neumaier-Probst E, et al. Local intra-arterial fibrinolysis in acute hemispheric stroke: effect of occlusion type and fibrinolytic agent on recanalization success and neurological outcome. Cerebrovasc Dis 2003;15:258-63.

23 Sorimachi T, Fujii $Y$, Tsuchiya $N$, et al. Recanalization by mechanical embolus disruption during intra-arterial thrombolysis in the carotid territory. AJNR Am J Neuroradiol 2004;25:1391-402.

24 Jovin TG, Gupta R, Uchino K, et al. Emergent stenting of extracranial internal carotid artery occlusion in acute stroke has a high revascularization rate. Stroke 2005;36:2426-30.

25 Nedeltchev K, Brekenfeld C, Remonda L, et al. Internal carotid artery stent implantation in 25 patients with acute stroke: preliminary results. Radiology 2005;237:1029-37.

26 Mori T, Izumoto $\mathrm{H}$, Takabatake $\mathrm{N}$, et al. Clot removal therapy by aspiration and extraction for acute embolic carotid occlusion. AJNR Am J Neuroradiol 2006;27:1521-7 
27 Suh DC, Kim JK, Choi CG, et al. Prognostic factors for neurologic outcome after endovascular revascularization of acute symptomatic occlusion of the internal carotid artery. AJNR Am J Neuroradiol 2007;28:1167-71.

28 Arnold M, Kappeler L, Nedeltchev K, et al. Recanalization and outcome after intra-arterial thrombolysis in middle cerebral artery and internal carotid artery occlusion: does sex matter? Stroke 2007;38:1281-5.

29 Kim DJ, Kim DI, Byun JS, et al. Intra-arterial thrombolytic therapy for hyperacute ischemic stroke caused by tandem occlusion. Cerebrovasc Dis 2008;26:184-9.

30 Miteff F, Faulder KC, Goh CC, et al. Mechanical thrombectomy with a self-expanding retrievable intracranial stent (Solitaire $A B$ ): experience in 26 patients with acute cerebral artery occlusion. AJNR Am J Neuroradiol 2011;32:1078-81.

31 Watanabe M, Mori T, Imai K, et al. Endovascular interventions for patients with serious symptoms caused by embolic carotid T occlusion. Neurol Med Chir 2011;51:282-8.

32 Fesl G, Wiesmann M, Patzig M, et al. Endovascular mechanical recanalisation of acute carotid-T occlusions: a single-center retrospective analysis. Cardiovasc Intervent Radiol 2011;34:280-6.

33 Papanagiotou P, Roth C, Walter $\mathrm{S}$, et al. Carotid artery stenting in acute stroke. J Am Coll Cardiol 2011;58:2363-9.

34 Malik AM, Vora N, Lin R, et al. Endovascular treatment of tandem extracranial/ intracranial anterior circulation occlusions: preliminary single-center experience. Stroke 2011:42:1653-7.

35 Hauck EF, Natarajan SK, Ohta H, et al. Emergent endovascular recanalization for cervical internal carotid artery occlusion in patients presenting with acute stroke. Neurosurgery 2011;69:899-907.
36 Hwang $\mathrm{Y}-\mathrm{H}$, Kang D-H, Kim Y-W, et al. Outcome of forced-suction thrombectomy in acute intracranial internal carotid occlusion. J Neurointerv Surg 2013;5:81-4.

37 Fischer U, Mono M-L, Schroth G, et al. Endovascular therapy in 201 patients with acute symptomatic occlusion of the internal carotid artery. Eur J Neurol 2013;20:1017-24.

38 Yoon YH, Yoon W, Jung MY, et al. Outcome of mechanical thrombectomy with Solitaire stent as first-line intra-arterial treatment in intracranial internal carotid artery occlusion. Neuroradiology 2013;55:999-1005.

39 Mpotsaris M, Bussmeyer M, Buchner $\mathrm{H}$, et al. Clinical outcome of neurointerventional emergency treatment of extra- or intracranial tandem occlusions in acute major stroke: antegrade approach with wallstent and solitaire stent retriever. Clin Neuroradiol 2013;23:207-15.

40 Lee JS, Hong JM, Lee S-J, et al. The combined use of mechanical thrombectomy devices is feasible for treating acute carotid terminus occlusion. Acta Neurochir 2013;155:635-41.

41 Matsubara N, Miyachi S, Tsukamoto N, et al. Endovascular intervention for acute cervical carotid artery occlusion. Acta Neurochir 2013;155:1115-23.

42 Kwak HS, Hwang SB, Jin GY, et al. Predictors of functional outcome after emergency carotid artery stenting and intra-arterial thrombolysis for treatment of acute stroke associated with obstruction of the proximal internal carotid artery and tandem downstream occlusion. AJNR Am J Neuroradiol 2013;34:841-6.

43 Nogueira RG, Lutsep HL, Gupta R, et al. Trevo versus Merci retrievers for thrombectomy revascularisation of large vessel occlusions in acute ischaemic stroke (TREVO 2): a randomised trial. Lancet 2012;380:1231-40.

44 Saver JL, Jahan R, Levy El, et al. Solitaire flow restoration device versus the Merci Retriever in patients with acute ischaemic stroke (SWIFT): a randomised, parallel-group, non-inferiority trial. Lancet 2012;380:1241-9. 